\title{
DEFINABLY CONNECTED NONCONNECTED SETS
}

\author{
ANTONGIULIO FORNASIERO
}

Abstract. We give an example of a structure $\mathrm{R}$ on the real line, and a manifold $\mathrm{M}$ definable in $\mathrm{R}$, such that $\mathrm{M}$ is definably connected but is not connected.

\section{INTRODUCTION}

Let $\mathcal{R}$ be an expansion of the real field $\overline{\mathbb{R}}:=\langle\mathbb{R},+, \cdot,<\rangle$. Let $M$ be a definable subset of $\mathcal{R}$. We remind that $M$ is called definably connected if there is no clopen definable subset $Y$ of $M$ such that $\emptyset \neq Y \neq M$.

Main Theorem. There exists a structure $\mathcal{R}$ expanding $\overline{\mathbb{R}}$ and set $M$ definable in $\mathcal{R}$, such that:

(1) $M$ is a 1-dimensional embedded $\mathcal{C}^{1}$ submanifold of $\mathbb{R}^{3}$,

(2) $M$ has 2 connected components,

(3) $M$ is definably connected.

We know that such $\mathcal{R}$ cannot be o-minimal, because in an o-minimal expansion of $\mathbb{R}$ every definable and definably connected set is arc-connected (and hence connected). However, we can find $\mathcal{R}$ as above which is also d-minimal (see [Mil05] for the definition and main properties of d-minimal structures).

The main ingredient is the following result, which follows easily from the proof of [MT06, Theorem 1]; we will give some details of the proof in $\S 3$.

Lemma 1.1. There exists a sequence $P=\left\langle c_{n}: n \in \mathbb{N}\right\rangle$ of real numbers, such that

(1) $P$ is strictly increasing and unbounded;

(2) the set $Q:=\left\{c_{n}: n\right.$ even $\}$ (as a set) is not definable in $\mathcal{R}$, the expansion of $\overline{\mathbb{R}}$ with a new predicate for $P$ (where $P$ is also regarded as a set).

Moreover, we can find $P$ as above such that $\mathcal{R}$ is also d-minimal.

We will show that $\mathcal{R}$ as in the above lemma satisfies the conclusion of the Main Theorem.

1.1. Application to Pfaffian functions. Let $\mathcal{R}$ and $M$ be as in the proof of the Main Theorem. In [Fra06], S. Fratarcangeli introduced the relative Pfaffian closure of $\overline{\mathbb{R}}$ inside $\mathcal{R}$. Consider the following 1 -form on $\mathbb{R}^{3} \omega(x, y, z):=d z$. Let $L$ be the $x y$-coordinate plane. Notice that $L$ is a Rolle Leaf with data $\left\langle\mathbb{R}^{2}, \omega\right\rangle$, according to the definition in [Spe99]. Let $X_{0}$ be the translate of $M$ along the $z$-axis, such that the endpoints of $M$ are on $L, X_{1}$ be its mirror image along the $x y$-plane, and $X:=X_{0} \cup X_{1}$. Then, $X$ is a 1 -dimensional $\mathcal{C}^{1}$ manifold which is definable in $\mathcal{R}$ and definably connected, which intersects $L$ in 2 points, but which is never orthogonal to $\omega$. Thus, $L$ is not a $\mathcal{R}$-Rolle Leaf, according to [Fra06, Definition 5.2]: therefore, it is not always the case that a Rolle Leaf (à la Speissegger) definable in $\mathcal{R}$ and with data definable in $\overline{\mathbb{R}}$ is a $\mathcal{R}$-Rolle Leaf à la Fratarcangeli.

Date: 18 Mar 2011.

2010 Mathematics Subject Classification. Primary 03Cxx; Secondary 12J15, 03C64.

Key words and phrases. Connected, definably connected.

Thanks to Immanuel Halupczok and Philipp Hieronymi for their help in writing this article. 


\section{Proof of the Main Theorem}

$\mathcal{R}$ is the structure introduced in Lemma 1.1. We have to produce the manifold $M$. Let $C_{0}$ be the double helix in $\mathbb{R}^{3}$ obtained as union of the helices

$$
\left\{\begin{array} { l } 
{ x = \operatorname { s i n } ( \theta ) } \\
{ y = \operatorname { c o s } ( \theta ) } \\
{ z = \theta / \pi , }
\end{array} \text { and } \quad \left\{\begin{array}{l}
x=-\sin (\theta) \\
y=-\cos (\theta) \\
z=\theta / \pi,
\end{array}\right.\right.
$$

with $0 \leq \theta \leq 1$. Let $C$ be an embedded 1-dimensional manifold definable in $\overline{\mathbb{R}}$, of the "same shape" as $C_{0}$, such that the endpoints of $C$ and $C_{0}$ coincide. What we mean is the following:

(1) $C$ is the union of 2 disjoint $\mathcal{C}^{1}$ arcs, $C_{1}$ and $C_{2}$, where $C_{1}$ is the graph of a $\mathcal{C}^{1}$ function $g_{1}:[0,1] \rightarrow[-1,1]^{2}$ (with domain the $z$-axis), and the same for $C_{2}$;

(2) $g_{1}(0)=\langle 0,1\rangle, g_{1}(1)=\langle 0,-1\rangle, g_{2}(0)=\langle 0,-1\rangle, g_{1}(1)=\langle 0,1\rangle$;

(3) $g_{i}(t) \in(-1,1)^{2}$ for $i=1,2$ and $t \in(0,1)$;

(4) We also ask that $\langle 0,0\rangle=g_{1}^{\prime}(0)=g_{1}^{\prime}(1)=g_{2}^{\prime}(0)=g_{2}^{\prime}(1)$ (notice that this latter condition is not satisfied by $\left.C_{0}\right)$.

For every $n \in N$, let $D_{n}$ be the double helix obtained from $C$ by translation and dilation along the $z$-axis, such that the lower endpoints of $D_{n}$ are $\left\langle 0, \pm 1, c_{n}\right\rangle$ and the upper endpoints are $\left\langle 0, \pm 1, c_{n+1}\right\rangle$. Finally, let $M:=\bigcup_{n} D_{n}$. We claim that $M$ satisfies the conclusion of the Main Theorem. The fact that $M$ is definable in $\mathcal{R}$ and has 2 connected components $M_{1}$ and $M_{2}$ is clear (where $M_{1}$ is the component containing the point $\left.\left\langle 0,1, d_{0}\right\rangle\right)$. The fact that $M$ is $\mathcal{C}^{1}$ is clear from (4). It remains to show that $M$ is definably connected. If not, then $M_{1}$ would be definable in $\mathcal{R}$. However, $\langle 0,1, z\rangle \in M_{1}$ iff $z \in Q$ : hence, $Q$ would be definable in $\mathcal{R}$, contradiction.

\section{Proof of Lemma 1.1}

In this section we sketch the proof of Lemma 1.1. Let $1<c \in \mathbb{R}$ and $P$ be the sequence $\left\{c^{2^{n}}: n \in \mathbb{N}\right\}$, and $\mathcal{R}:=\overline{\mathbb{R}}(P)$. By [MT06, Corollary 7], $\mathcal{R}$ is d-minimal. In the proof of [MT06, Theorem 1], Miller and Tyne establish the following result.

Fact 3.1. Let $\overline{\mathcal{L}}:=\langle 0,1,+, \cdot,\langle\rangle$ be the language of ordered fields, and $\overline{\mathcal{L}}(P)$ be its extension by the new unary predicate $P$ (thus, $\mathcal{R}$ is naturally an $\overline{\mathcal{L}}(P)$-structure). Let $\left\langle\mathbb{F}, P^{*}\right\rangle$ be an elementary extension of $\langle\overline{\mathbb{R}}, P\rangle$. Let $b$ and $b^{\prime}$ be in $P^{*}$, such that $b$ and $b^{\prime}$ be in $Q$ such that satisfy the same $\overline{\mathcal{L}}$-type over $\mathbb{R}$. Then, $b$ and $b^{\prime}$ satisfy the same $\overline{\mathcal{L}}(P)$-type over $\mathbb{R}$.

Assume now, by contradiction, that $Q$, the subset of $P$ given by the elements with even index, is definable in $\mathcal{R}$, by an $\overline{\mathcal{L}}(P)$-formula (with parameters from $\mathbb{R}$ ) $\phi(x)$. Let $\left\langle\mathbb{F}, P^{*}\right\rangle$ be an $\omega$-saturated elementary extension of $\mathcal{R}$. Let $b$ be in $P^{*}$, such that $b>\mathbb{R}$ and $\phi(b)$ holds. Let $b^{\prime}$ be the successor of $b$ in $P^{*}$. Then, $\neg \phi\left(b^{\prime}\right)$ holds. However, $b$ and $b^{\prime}$ satisfy the same $\overline{\mathcal{L}}$-type over $\mathbb{R}$, contradicting Fact 3.1 .

\section{REFERENCES}

[Fra06] Sergio Fratarcangeli, Rolle leaves and o-minimal structures, Ph.D. Thesis, McMaster University, April 2006. $\uparrow 1$

[Mil05] Chris Miller, Tameness in expansions of the real field, Logic Colloquium '01, Lect. Notes Log., vol. 20, Assoc. Symbol. Logic, Urbana, IL, 2005, pp. 281-316. MR2143901 (2006j:03049) $\uparrow 1$

[MT06] Chris Miller and James Tyne, Expansions of o-minimal structures by iteration sequences, Notre Dame J. Formal Logic 47 (2006), no. 1, 93-99, DOI 10.1305/ndjfl/1143468314. MR2211185 (2006m:03065) $\uparrow 1,2$

[Spe99] Patrick Speissegger, The Pfaffian closure of an o-minimal structure, J. Reine Angew. Math. 508 (1999), 189-211, DOI 10.1515/crll.1999.026. MR1676876 (2000j:14093) $\uparrow 1$ 
Institut für Mathematische Logik, Einsteinstr. 62, 48149 Münster, Germany URL: http://www.dm.unipi.it/ fornasiero/

E-mail address: antongiulio.fornasiero@googlemail.com 